\title{
Globose, cystic olfactory ensheathing cell tumor: A case report and literature review
}

\author{
YAOLING LIU, MINGHAI WEI, KANG YANG, ZESHI TAN, XU SUN, XINYU LI, NINGWEI CHE, \\ LAN LUAN, GUANYU WANG, XIAOFENG WANG, YUQIANG SUN and JIAN YIN \\ Department of Neurosurgery, The Second Affiliated Hospital of Dalian Medical University, \\ Dalian, Liaoning 116044, P.R. China
}

Received February 4, 2015; Accepted March 15, 2016

DOI: $10.3892 / \mathrm{ol} .2016 .5131$

\begin{abstract}
Olfactory ensheathing cell tumor (OECT) is one of the most rare intracranial, extra-axial tumors located in the anterior cranial fossa. The present study reports a case of a 34-year-old female patient who presented with a history of hyposmia for 1 year, as well as a gradual dizziness and emotional lability for 2 months. Magnetic resonance imaging of the brain revealed a globose, well-defined cystic mass at the midline of the anterior cranial fossa, which was confirmed as an OECT by histology and was completely resected by bifrontal craniotomy. According to the immunostaining results, the tumor was positive for vimentin and S100 protein, and negative for epithelial membrane antigen, glial fibrillary acidic protein and cluster of differentiation 57 (also known as Leu-7). The presentation, imaging findings, histopathological examination and histogenesis of OECT are discussed in the present study, along with a literature review.
\end{abstract}

\section{Introduction}

Traditional theories have supported that schwannomas originate from the optic and olfactory nerve, which, however, is rather unlikely, since these two nerves do not have a Schwann cell layer (1). Olfactory ensheathing cell tumor (OECT) is considered to originate from OECs of the olfactory mucosa or bulb. Not until Yasuda et al reported a case of OECT in 2006 did researchers start to focus on this rare type of tumor (2). Despite its rarity, $\sim 50$ cases of subfrontal olfactory groove schwannoma (OGS) and 8 cases of OECT, with clinical and

Correspondence to: Dr Jian Yin or Dr Minghai Wei, Department of Neurosurgery, The Second Affiliated Hospital of Dalian Medical University, 467 Zhongshan Road, Shahekou, Dalian, Liaoning 116044, P.R. China

E-mail: dyeyDr_yin@163.com

E-mail: WMH_dyey@163.com

Key words: olfactory groove schwannoma, olfactory ensheathing cells, olfactory ensheathing cell tumor, Leu-7 (cluster of differentiation 57), olfactory groove meningioma radiological features similar to those of meningiomas and neuroblastomas in the midline of the anterior cranial fossa, have been published worldwide to date (2-11). The biological behaviour of OECT is currently unknown; The previous cases reported a benign course and a short follow-up time (2,5-11). OECs resemble Schwann cells on light and electron microscopy; however, the presence of cluster of differentiation 57, also known as Leu-7, is considered to differentiate OGS from OECT (2). OECT may be treated surgically using the endoscopic transnasal approach or craniotomy. OECT has an extremely good prognosis, with a reported 2-year survival rate of $100 \%(2,5-11)$. The present study reports the ninth case of OECT, which included confusing radiographic features, and presents a review of the literature.

\section{Case report}

On March 10, 2014, a 34-year-old woman was admitted to The Second Affiliated Hospital of Dalian Medical University (Dalian, China) presenting with a history of hyposmia for 1 year, accompanied by a gradual dizziness and emotional lability for 2 months. No seizures, visual disorder, cerebrospinal fluid rhinorrhea or neurofibromatosis-related family history was recorded. Physical examination detected neither focal neurological deficits nor abnormal pigmentation of neurofibromatosis, with the exception of right hyposmia. Magnetic resonance imaging (MRI) scan (MR Signa 3.0T Excite; GE Healthcare Bio-Sciences, Pittsburgh, PA, USA) revealed a 3.0x3.0x3.1-cm extra-axial, globose, well-defined mass at the midline of the anterior cranial fossa, which deviated to the right. The tumor displayed homogeneous hypointensity on T1-weighted images, hyperintensity on T2-weighted images and isointensity on fluid-attenuated inversion recovery, and was shown to have caused brain parenchyma deformation without obvious peritumoral edema (Fig. 1A, B and C). Following the administration of intravenous gadolinium (Sigma-Aldrich, St. Louis, MO, USA), the tumor was heterogeneously enhanced and a peculiar membrane was observed inside the cystic wall. The tumor boundary appeared clear and smooth, while the main part was tightly connected to the endocranium (Fig. 1D and $\mathrm{E}$ ).

The patient underwent bifrontal craniotomy. When the right frontal lobe was softly lifted, a grayish red tumor with 
A

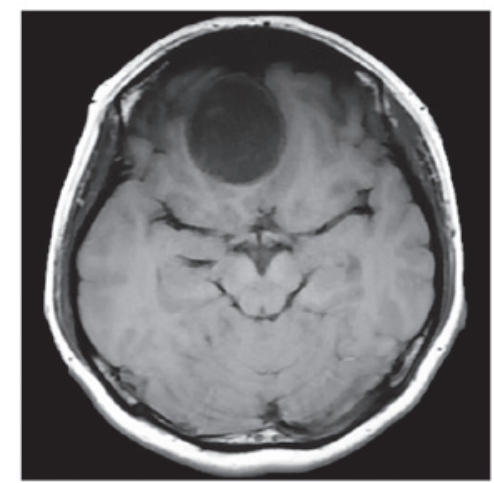

D

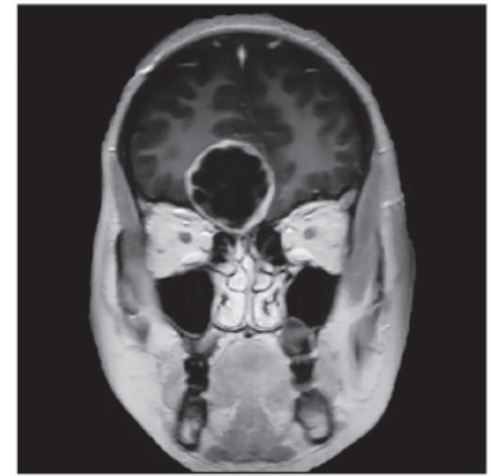

B

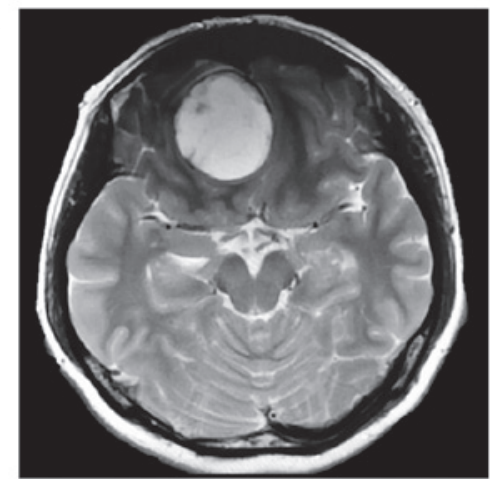

$\mathbf{E}$

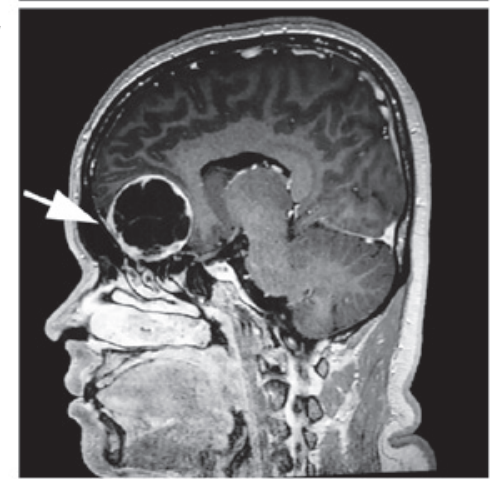

$\mathrm{C}$

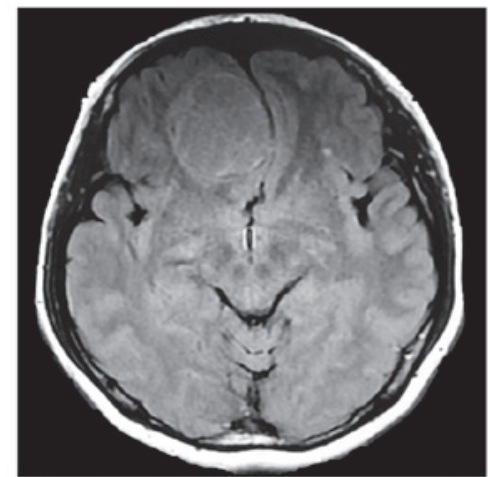

F

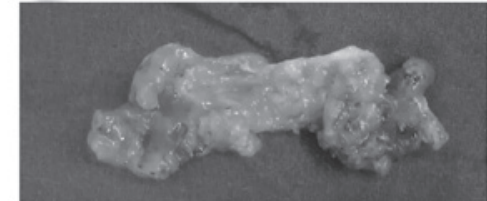

Figure 1. MRI of the brain. (A) Homogeneous hypointensity was observed on T1-weighted images (axial view). (B) Homogeneous hyperintensity was observed on T2-weighted images (axial view). (C) Homogeneous isointensity was observed on fluid-attenuated inversion recovery image (axial view). (D) Enhanced MRI on coronal view showing that the bone of the frontal base was slightly depressed toward the nasal cavity. (E) MRI scan showing that the basilar part of the tumor was tightly connected to the endocranium, mimicking dural tail sign on sagittal view (arrow). (F) Top: The tumor appeared fat-like inside upon dissection, as it was yellow and soft. Bottom: The tumor was covered by a smooth capsule. MRI, magnetic resonance imaging.

a glistening appearance was observed to be attached to the olfactory groove. The tumor was located in the intradural, extra-axial space, attached to the right anterior part of the cribriform plate. A large volume of a clear, non-congealable yellow liquid was extracted from inside the tumor. In addition, the right olfactory tract could not be identified, and the left olfactory tract had been squeezed by the tumor. The tumor was completely resected, and when dissected, it appeared to contain fat, as it was soft and yellow (Fig. 1F), however subsequent pathology revealed that fat was not present. The cribriform plate protruded slightly towards the nasal cavity; however, the bone cortex was intact. Using a microscope (MT4000D; Meiji Seika Kaisha, Ltd., Tokyo, Japan), the tumor tissue was examined histologically and the findings described a tumor composed of spindle cells, with an eosinophilic protoplasm and tadpole-shaped nucleus. No tumor necrosis or blood vessel hyperplasia was observed (Fig. 2A). Immunostaining results revealed positivity for $\mathrm{S} 100$ protein (polyclonal rabbit anti-human S100 antibody; cat. no. ENT4197; 1:10; Elabscience Biotechnology Co., Ltd, Wuhan, China) (Fig. 2B) and vimentin (polyclonal rabbit anti-human antibody; cat. no. ENT4879; 1:10; Elabscience Biotechnology Co., Ltd.) (Fig. 2C), and negativity for epithelial membrane antigen (monoclonal rat anti-human antibody; cat. no. BM0042; 1:10; Boster Inc., Wuhan, China) (Fig. 2D), glial fibrillary acidic protein (polyclonal chicken anti-mouse cat. no. ab4674; 1:10; Abcam, Cambridge, MA, USA) (Fig. 2E) and Leu-7 (monoclonal mouse anti-human antibody; cat. no. ab187274; 1:10; Abcam) (Fig. 2F). The Ki-67 (polyclonal rabbit anti-human antibody; cat. no. EPP14636; 1:10; Elabscience Biotechnology Co., Ltd.) index was $1 \%$
(Fig. 2G); therefore, the final pathological diagnosis was OECT. The postoperative course of the patient was uneventful, without adjuvant radiation and chemotherapy. No evidence of tumor recurrence was observed during the 6-month radiographic follow-ups.

\section{Discussion}

A literature search for relevant publications was performed in the followingdatabases:PubMed(www.ncbi.nlm.nih.gov/pubmed), Web of Science (http://isiknowledge.com), MEDLINE (http://ovidsp.ovid.com/), Excerpta Medica dataBASE (www.elsevier.com/solutions/embase-biomedical-research) and Chinese Biomedical Database (www.sinomed.ac.cn/zh/), using the keywords 'olfactory ensheathing cell tumor' and 'olfactory groove schwannoma' in titles and/or abstracts. The search identified 8 cases of OECT (Table I). A review of these 8 cases in addition to the present one revealed that the initial symptoms of the patients included hyposmia or anosmia (6/7 patients; $86 \%$; initial symptoms were not reported in 2 cases), seizures (4/9 patients; 44\%), emotional lability (2/9 patients; $22 \%$ ), headache and dizziness (3/9 patients; $33 \%$ ), visual impairment (1/9 patients; $11 \%)$ and foreign body sensation (1/9 patients; $11 \%)$. The studies did not mention whether the patients that suffered the seizures were suspected of having OECT. A possible explanation for the seizures is dualism, since OECT is an extra-axial tumor, which makes it less likely for patients to develop seizures and thus, OECT and seizures may be derived from two separate pathologies. The mean age of the 9 patients reviewed was 31.9 years, which was consistent 


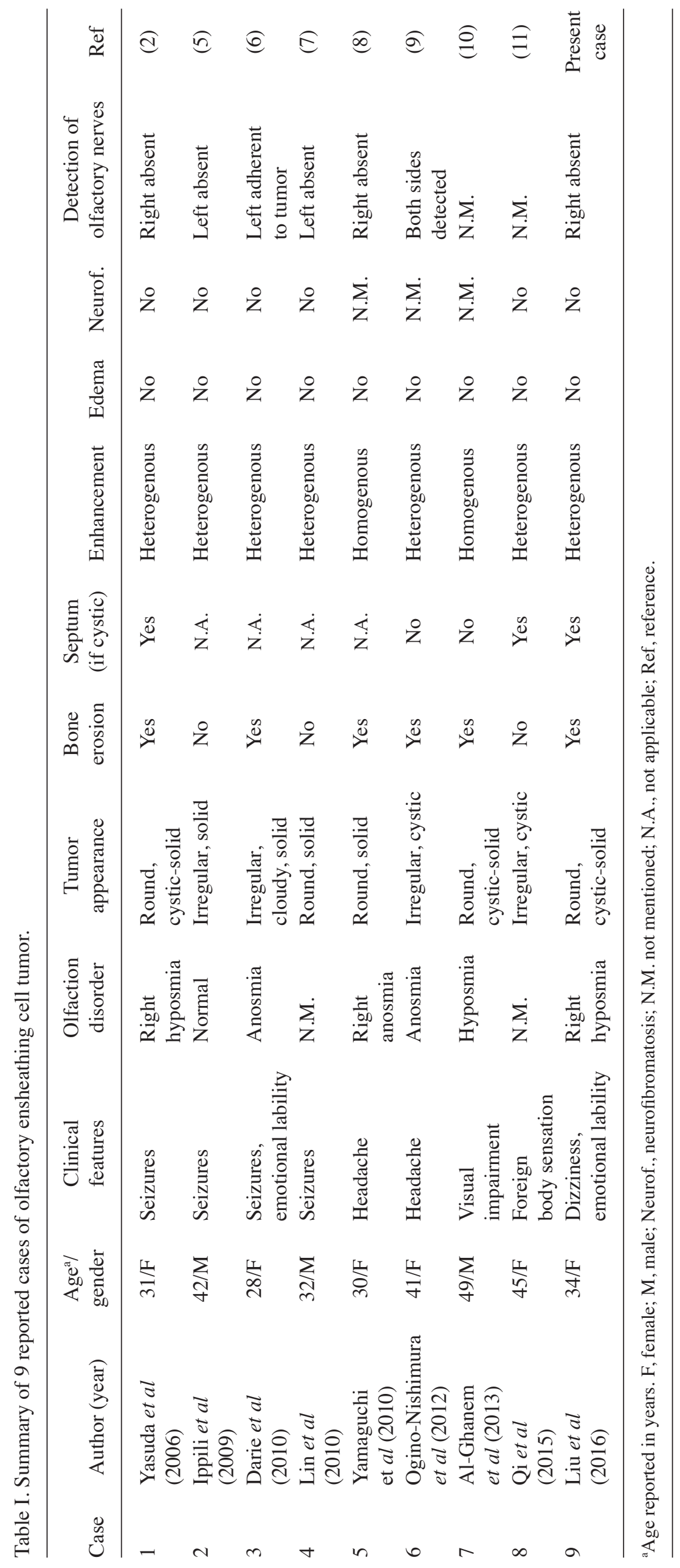


A

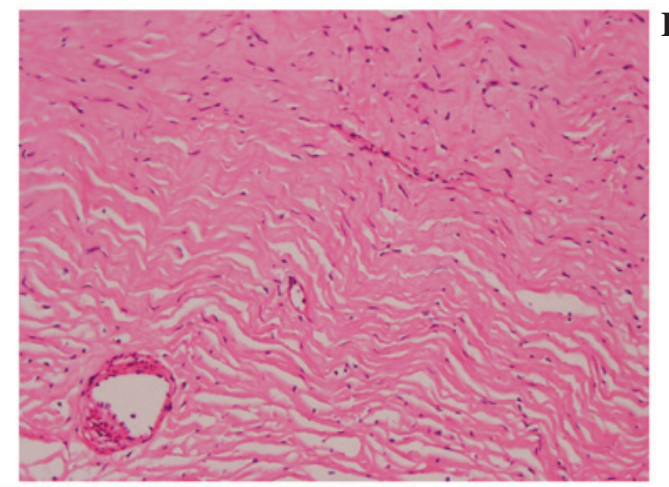

C

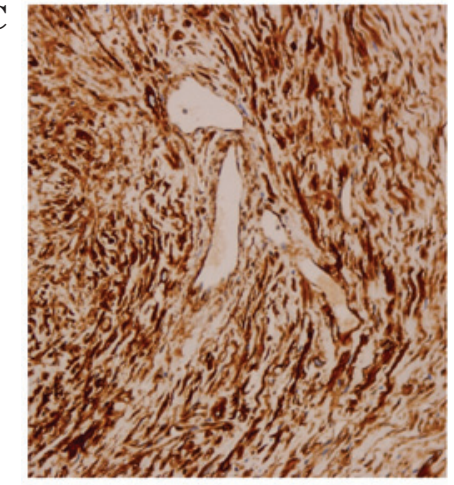

F

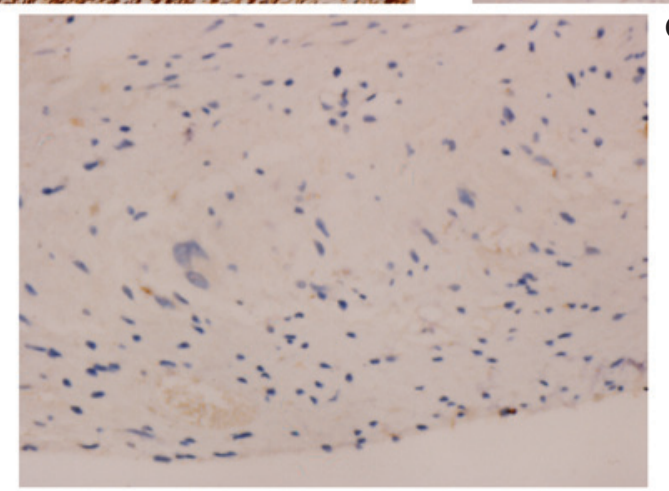

D
B
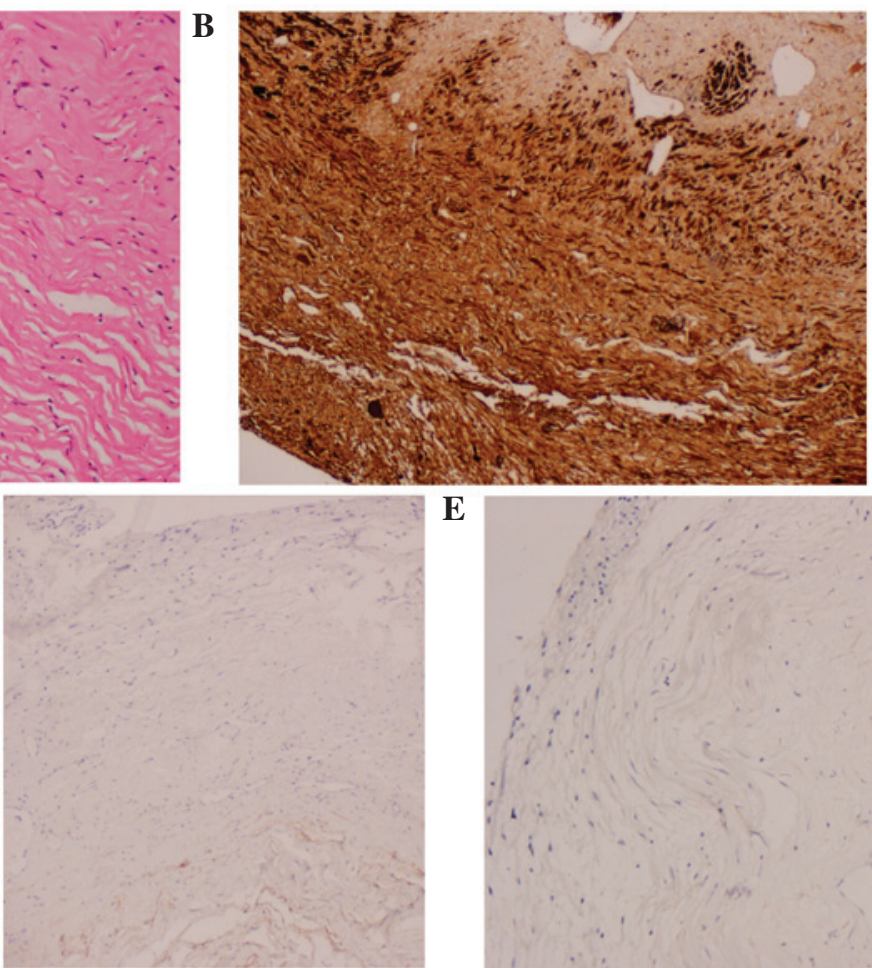

E
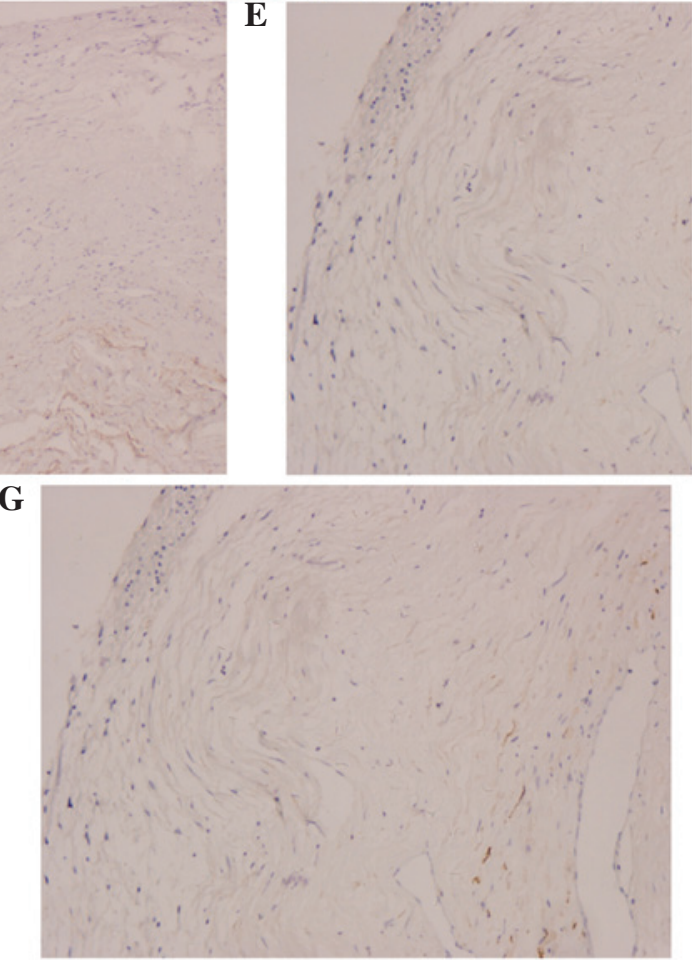

Figure 2. Histopathological and immunostaining results. (A) Hematoxylin and eosin staining (magnification, $\mathrm{x} 40$ ). The tumor was positive for (B) S100 (magnification, $\mathrm{x} 40$ ) and (C) vimentin (magnification, $\mathrm{x} 40$ ), and negative for (D) epithelial membrane antigen (magnification, x40), (E) glial fibrillary acidic protein (magnification, $\mathrm{x} 40$ ) and (G) Leu-7 (magnification, $\mathrm{x} 40$ ). (F) The Ki-67 index was 1\% (magnification, x100).

with that of patients with OGS $(32.7 \pm 14.0$ years) $(12)$, and a female predominance was observed (females, 67\%; males, $33 \%)$. In addition, radiographic evaluation revealed that 2/9 OECTs $(22 \%)$ were cystic, 3/9 (33\%) cystic-solid and $4 / 9(44 \%)$ solid. Of note, the proportion of septum in cystic tumors was $60 \%(n=3 / 5)$, which was quite different from the majority of the cystic OGSs. If further studies confirm this finding, it may serve as an important radiographic clue for the diagnosis of OECTs. Furthermore, 7/9 OECTs (78\%) were heterogeneously enhanced, while 2/9 (22\%) were homogeneously enhanced. Bone erosion was a rather common finding (6/9 cases; $67 \%$ ), whereas none of the patients presented with peritumoral edema. Intraoperative findings revealed that, out of the 9 OECTs, 8 originated from the olfactory bulb or tract, and 1 originated from the olfactory mucosa (9).

OECs are specialized glial cells capable of migrating even in astrocyte-rich environments; they ensheathe olfactory axons in order to facilitate axonal growth. Due to these characteristics, OECs have attracted considerable attention $(11,13)$. Notably, OECs resemble Schwann cells in their appearance on light and electron microscopy, and in the majority of immunohistochemical staining features. The differentiation between OGS and OECT is mainly based on the presence of Leu-7 $(2,5,14)$. All the cases of OECT reviewed in the present study were negative for Leu-7 (2,5-11); however, in the majority of OGS cases, testing for Leu-7 was either not performed or not mentioned in the corresponding studies $(4,12,15)$. Furthermore, information on the Leu-7 reactivity in OGS is limited; $\sim 20 \%$ of the tumors that were considered to be schwannomas were negative for Leu-7 $(9,16)$; therefore, certain reported cases of OGS could have originated from OECs rather than Schwann cells.

In the present case, the radiological manifestations of the OECT were confusing, as a dural tail sign was evident on enhanced MRI and bone sclerosis and calcification were identified on computed tomography, which are similar to those observed with OGM (9). The differential diagnosis of tumors involving the extra-axial anterior cranial fossa, with or without bulging into the ethmoid sinus, should include OGM, OGS, OECT, 


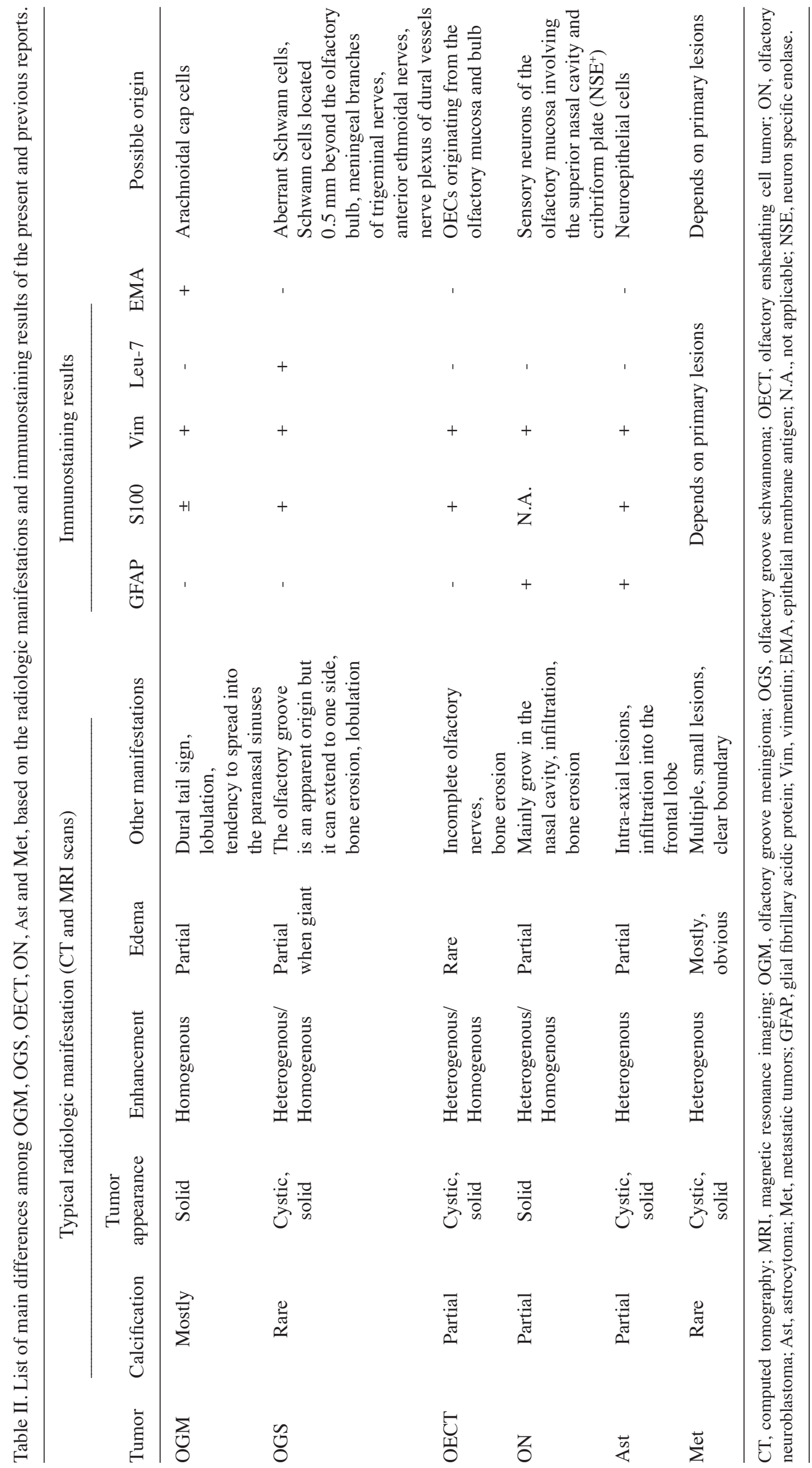


low-grade astrocytoma, olfactory neuroblastoma and metastatic tumors. In the present case, the extra-axial tumor with the clear boundary that was observed to be connected to the endocranium, could have potentially been misdiagnosed as a dural tail sign on sagittal view MRI following contrast-enhancement. The presence of bone scalloping, as well as the absence of bone sclerosis and calcification, may assist the differentiation between OECTs and meningiomas (17). By contrast, OGMs exhibit a propensity to spread into the paranasal sinuses, particularly in recurrent cases, without displaying evidence of calcification. Furthermore, multiple foci of T2-hypointense MRI signals (associated with microbleeds) may suggest schwannoma (5). Table II comprises a list of the main differences among OGM, OGS, OECT, low-grade astrocytoma, olfactory neuroblastoma and metastatic tumors, based on radiologic manifestations, immunostaining results and possible origin.

In previous reports, the aforementioned conditions were misdiagnosed as each other preoperatively, due to their rarity and enigmatic origin. The embryonic development of OEC is olfactory placode-derived, while that of Schwann cells is neural crest-derived; therefore, both tumors possess a similar peripheral origin (1). The following five main origins have been reported for OGS to date: i) Aberrant Schwann cells; ii) Schwann cells located $0.5 \mathrm{~mm}$ beyond the olfactory bulb; iii) meningeal branches of trigeminal nerves; iv) nerve plexus of dural vessels; and v) anterior ethmoidal nerves (4). Glial cells originate from the central nervous system (CNS), however, OECT has been demonstrated to originate from OECs of the olfactory mucosa or bulb, which are part of the peripheral nervous system (PNS). Thus, OECs may be considered an intermediate glial cell type that originate in the CNS, but function in the PNS. Furthermore, olfactory neuroblastoma has been reported to originate from the sensory neurons of the olfactory mucosa, and to involve the superior nasal cavity or the cribriform plate, while OGM has been reported to originate from arachnoidal cap cells as a truism $(7,18)$.

Although the reported cases experienced a benign course, the follow-up for these cases are too short, and the origin of OECT, its biological behavior and the necessity for adjuvant chemotherapy remains uncertain. In conclusion, according to the limited number of clinical cases of OECT reported in the literature thus far, total surgical removal of the tumor remains the suggested treatment option, and long-term follow-up appears to be required.

\section{References}

1. Wewetzer K, Verdú E, Angelov DN and Navarro X: Olfactory ensheathing glia and Schwann cells: Two of a kind? Cell Tissue Res 309: 337-345, 2002.

2. Yasuda M, Higuchi O, Takano S and Matsumura A: Olfactory ensheathing cell tumor: A case report. J Neurooncol 76: 111-113, 2006.

3. Salunke P, Patra DP, Futane S and Nada R: Olfactory region schwannoma: Excision with preservation of olfaction. J Neurosci Rural Pract 5: 281-283, 2014.

4. Figueiredo EG, Soga Y, Amorim RL, Oliveira AM and Teixeira MJ: The puzzling olfactory groove schwannoma: A systematic review. Skull Base 21: 31-36, 2011.

5. Ippili K, Ratnam BG, Gowrishankar S, Ranjan A and Lath R: Olfactory ensheathing cell tumor. Neurol India 57: 76-78, 2009.

6. Darie I, Riffaud L, Saïkali S, Brassier G and Hamlat A: Olfactory ensheathing cell tumour: Case report and literature review. J Neurooncol 100: 285-289, 2010.

7. Lin SC, Chen MH, Lin CF and Ho DM: Olfactory ensheathing cell tumor with neurofibroma-like features: A case report and review of the literature. J Neurooncol 97: 117-122, 2010.

8. Yamaguchi T, Fujii H, Dziurzynski K, Delashaw JB and Watanabe E: Olfactory ensheathing cell tumor: Case report. Skull Base 20: 357-361, 2010.

9. Ogino-Nishimura E, Nakagawa T, Mikami Y and Ito J: Olfactory ensheathing cell tumor arising from the olfactory mucosa. Case Rep Med 2012: 426853, 2012.

10. Al-Ghanem R, Ramos-Pleguezuelos FM, Pérez-Darosa SI, Galicia-Bulnes JM, Cabrerizo-Carvajal F and El-Rubaidi OA: Olfactory ensheathing cell tumour: Case report and literature review. Neurocirugia (Astur) 24: 130-134, 2013 (In Spanish).

11. Qi X, Wan Y, Yan Q, Wang Y and Yang S: Cystic olfactory ensheathing cell tumor: A case report. Acta Neurol Belg 115: 191-193, 2015.

12. Li YP, Jiang S, Zhou PZ and Ni YB: Solitary olfactory schwannoma without olfactory dysfunction: A new case report and literature review. Neurol Sci 33: 137-142, 2012.

13. Vincent AJ, Taylor JM, Choi-Lundberg DL, West AK and Chuah MI: Genetic expression profile of olfactory ensheathing cells is distinct from that of Schwann cells and astrocytes. Glia 51: 132-147, 2005.

14. Bianco JI, Perry C, Harkin DG, Mackay-Sim A and Féron F: Neurotrophin 3 promotes purification and proliferation of olfactory ensheathing cells from human nose. Glia 45: 111-123, 2004.

15. Wang Z, Zhang W, You G, Wang J, Li G, Gao Z and Xie J: Olfactory schwannoma: A report of two cases and literature review. Neurol India 62: 429-431, 2014.

16. Johnson MD, Glick AD and Davis BW: Immunohistochemical evaluation of Leu-7, myelin basic-protein, S100-protein, glial-fibrillary acidic-protein, and LN3 immunoreactivity in nerve sheath tumors and sarcomas. Arch Pathol Lab Med 112: 155-160, 1988.

17. Santhosh K, Kesavadas C, Radhakrishnan VV, Thomas B, Kapilamoorthy TR and Gupta AK: Usefulness of T2*-weighted MR sequence for the diagnosis of subfrontal schwannoma. J Neuroradiol 34: 330-333, 2007.

18. Yamahata H, Hirahara K, Tomosugi T, Yamada M, Ishii T, Ishigami T, Uetsuhara K, Sueyoshi K, Matsukida S, Yatsushiro K and Arita K: Subfrontal schwannoma mimicking neuroblastoma: Case report. Skull Base Rep 1: 59-64, 2011 